\title{
PLANETARY TRANSMISSION LOAD SHARING: MANUFACTURING ERRORS AND SYSTEM CONFIGURATION STUDY
}

\author{
M. Iglesias, A. Fernandez del Rincon, A. de-Juan, \\ P. Garcia, A. Diez-Ibarbia, F. Viadero \\ Department of Structural and Mechanical Engineering, University of Cantabria. Avda. \\ de los Castros s/n 39005 Santander, Spain.
}

\begin{abstract}
This paper addresses the effect of manufacturing errors such as eccentricity and planet pin positioning errors on the quasi-static behavior of a 3 planet planetary transmission, taking into account different configurations regarding the bearing condition of the sun gear shaft. The aim of the paper is to shed light on some untouched aspects of the load sharing behavior of planetary transmissions, such as the effect of radial positioning errors of the planets when different pressure angles are used, and the impact of the different loadings per planet on the actual load per tooth.

A modeling approach is employed, and physical explanations and simplified graphs are provided to help understand the behavior of the transmission when the sun is allowed to float and errors are introduced. The model used, developed by the authors and presented and validated in previous works, hybridizes analytical solutions with finite element models in order to compute the contact forces.

The results obtained show that the teeth loads are much lower than expected compared to the planet uneven loads, both in the non-defected and defected transmission, and that radial positioning errors have non-negligible effect on the load sharing ratio under certain operating conditions.
\end{abstract}

Keywords: Gears, Planetary transmissions, Load sharing ratio, Transmission error, Run-out, Manufacturing errors 


\section{Introduction}

One of the main advantages of planetary transmissions is its compactness. For high torques, instead of enlarging the wheels and thus its load capacity, planetary transmissions split the load into a number of paths. In this manner, the power is divided among several pinions, so that loadings per unit facewidth remain below nominal values while the torque is multiplied. Besides, planetary transmissions present coaxial input and output and large reduction ratios, being the most compact and lightest possible drives [1]. Under ideal conditions, each path in a planetary transmission carries an equal amount of load. Nevertheless, as in real systems there are inevitable manufacturing deviations due to errors and tolerances, the load is not equally shared amongst the different sun/planet/ring meshes, which can be a problem in terms of both durability (higher loadings per unit facewidth than expected) and dynamic behaviour (vibrations due to changing loads, etc).

The load sharing problem in planetary transmissions has been discussed in a number of publications, assessed by means of experimental tests [2, 3], but mainly based on transmission modeling, from simpler analytical models [4] to more complex models including hybrid semi-analytical and finite element techniques [5].

Due to its spacial configuration, planetary transmissions are complicated to model, but the critical importance of these gear systems in aerospace and energy generation applications makes the effort worth it. The main feature that characterizes the dynamic behavior of gear transmissions is the change in the number of teeth couples simultaneously in mesh. The meshing stiffness is therefore variable, and induces a periodic excitation in the system. Thus, the characterization of this periodic excitation is crucial in order to achieve better simulated results [6]. In a first step to increase modeling realism, the static transmission error has been used as excitation to predict dynamic behavior of planetary transmissions [7, 8, 9]. Nevertheless, more recent studies point that this approach, whilst remaining relatively valid for ordinary transmissions, may not be applicable to multi-mesh transmissions such as planetary ones [10]. With a higher degree of accuracy, at a second step evolution, there are gear models with time-varying stiffness. They give better off-resonance responses, but they are also used to identify regions of large amplitude vibration near resonances, where damping and other nonlinear phenomena strongly affect the behavior $[11,12,13]$. The latest and more advanced planetary transmission models are those based on computa- 
tional approaches, frequently including FEM techniques in combination with different contact models [14]. In some cases, completely flexible bodies are considered in real time simulation [15]. Depending on the particular application of the model, different emphasize is given to each modeling aspect [16], as is the case of non-stationary operation [17].

Studies on load sharing have usually been focus on the behavior of the transmission when defects are present, evaluating the effect of different configurations on the resulting load sharing, trying to find methods of improvement. As latest works, in [18] the effects of gravity, ring support stiffness and bedplate tilt angle of a wind turbine on the load sharing is studied through modeling approaches. In [19] and again in the wind turbine field the load sharing behaviour of a compound planetary gear transmission in presence of multiple-errors is analysed, adding experimental results to verify the model approach. The effect of floating the sun gear in a planetary gearbox has been studied by [20], in order to absorb the consequences of geometrical imperfections.

In this paper, a planetary model is used to study the load sharing in quasi-static conditions, with the aim of shedding light on some untouched aspects of the load sharing behaviour of 3 planet planetary transmissions, such as the effect of radial positioning errors of the planets when different pressure angles are used and the impact of the different loadings per planet on the actual load per tooth. Specially in this last case, the new information can improve the understanding of the tooth load per unit length when uneven LSR occurs, and therefore to produce better gear design processes. Although this new design insight is a direct consequence of the study carried out, the ultimate goal of the planetary gear modeling research presented here is the accurate reproduction of the transmission behaviour in real conditions for on-condition monitoring assessment. The model used hybridizes analytical solutions with finite element models in order to compute the contact forces, making unnecessary the use of mesh stiffness waveform approximations or static transmission error excitation assumptions. The mesh model is based on previous work by the authors [21,22], extended and improved towards the planetary modeling as it can be found in [23]. Coupling through gear body deformations is also given a special attention, due to the multiple meshes per wheel. With respect to the contact point location and geometric overlap modeling approach used in this work, it has been conceived to allow for the almost direct inclusion of additional modeling features, such as tooth profile modifications (with an approach used in [24]) or the use of shifted gears [25]. 


\section{Planet load sharing}

There are many variations of planetary gear trains. However, whether simple or compound, with straight or helical gears, the vast majority of planetary transmissions designs share a fundamental quality: their compactness. This compactness can be understood in two different ways. The first has to do with the kinematic configuration of the planetary transmission (with a rotary planet carrier), which provides much higher ratios than those provided by conventional transmissions. Additionally, this configuration allows coaxial inputs and outputs, which is a plus for many applications, also economizing space. The second reason for which a planetary transmission can be seen as compact is the load capacity. The load capacity of a gear is ultimately determined by the size of their teeth, so that, in general, a large workload necessarily implies large gears accordingly. However, as planetary transmissions divide the total load on a variable number of paths (sun-planet and planetring pairs), the size of the gears can be reduced in the same proportion as the number of load paths used with respect to an ordinary transmission.

Ideally, each of the planetary load paths should transmit the same fraction of the total transmitted load. However, there are a number of reasons for which the load distribution may not be even in the actual operation of the planetary transmissions, the main two are the different path stiffness and the errors in the manufacturing and assembly process. Thus, there will be fluctuations in the working conditions of the various components, running out of the design conditions and causing overloads, in addition to the expected consequences on the dynamic behaviour.

Being this uneven load sharing among the different paths highly undesirable, the first of the two sources mentioned above could be easily avoided. The variable meshing stiffness is an inherent characteristic of gear transmissions. An unbalanced load share among the planetary paths can be caused by the different phase of the meshing cycle between paths, and the direct way to avoid this would be the synchronization of the meshing paths. When this design is adopted, another problem arises: the differences between simple and double contact for each mesh of each path would pile up in the complete transmission error or apparent stiffness of the total planetary transmission, magnifying their peak-to-peak fluctuation and becoming a great source of vibration and noise. Because of this, the choice of synchronized planet design is not usual and, on the contrary, a softened shape of the global transmission error signal is desired, for which the different meshing paths are phased out 
$2 \pi / n$ (where $n$ is the number of paths or planets).

With respect to the second source of uneven load sharing it is not easily avoidable, as it is related to all the errors in the manufacture or assembly of the various components of the planetary transmission. The present work focuses on these and particularly on the errors in the planet positioning.

As stated, the uneven load sharing has consequences both in the dynamic behavior: vibrations due to excitation by varying forces, and durability: higher workloads than nominal, or worse fatigue behavior of bearings and wheels due to the increase in the stresses fluctuation amplitude. That is why engineers and researchers have dedicated a great deal of attention to the study of planetary gears and the design of techniques to improve the load sharing. The most complicated solutions include flexible supports of the planets, specifically designed to absorb manufacturing or assembly errors. Another possible approach is to introduce flexible rings, also capable of suffering deflections which accommodate and absorb the causes of uneven load sharing. However, the simplest solution to achieve an improved load distribution is to allow one of the central members of the transmission to move freely, without any bearing restrictions on its movement around the nominal position. The axisymmetric spatial configuration of the planetary transmission allows to contemplate this solution, causing the central members to present a theoretical null radial load, and thus allowing the central elements to support themselves by effect of the combination of forces engaging on them, without conventional bearing support.

In this work, the planet load sharing has been defined as the ratio of the meshing torque in the sun due to each of the 3 planet-sun meshes, to the total input torque $T_{\text {ext }}$ calculated as:

$$
L S R_{i}=\frac{T_{(P i-S)}}{T_{\text {ext }}} ; \quad i=1,2,3
$$

Representing the relationship between the torque transmitted by the sun to each load paths and the total external torque applied. Therefore, in a three planet system as the used for these paper examples, the perfect Load Sharing Ratio (LSR) would be $1 / 3$. In the following sections, the real value of the LSR for each planet will be discussed, attending to the configuration of the system, the pin point positioning error of the planets in the tangential $\left(e_{t a n}\right)$ and in the radial $\left(e_{\text {rad }}\right)$ directions, and also paying attention to the role played by the magnitude of the torque to be transmitted (load level). 


\section{Planetary transmission configurations}

The study of the behavior of the planetary transmission presented in this paper is focused on the load sharing. With the aim of analyzing the influence of the transmission configuration on the load sharing behavior two different configurations are used in the study: fixed or floating sun, with the rest of elements constrained to strict rotation. Thus, in the case of the fixed sun configuration, the static equilibrium is defined for each position marked by the angular positions of the ring and planet carrier. Hence, the only degrees of freedom left to determine are the angular positions of planets and sun, and so four equilibrium equations are needed:

$$
\begin{aligned}
T_{S-P i}+T_{R-P i} & =0 \\
\sum_{i=1}^{3}\left(T_{S-P i}\right) & =T_{e x t}
\end{aligned}
$$

When considering the floating sun configuration, it is also necessary to determine the position of the sun gear, so the system of equations presented above must be extended with the balance of forces in the sun as:

$$
\sum_{i=1}^{3}\left(\mathbf{F}_{S-P i}\right)=\mathbf{0}
$$

As example, a real gear planetary reducer from agricultural machinery is modeled, whose main parameters are shown in Table 1 . The real application consists of two stages with a common ring, reason for which the ring width is larger than the rest of the wheels. All the results produced in this paper have been obtained from the model published in [23], which hybridizes analytical solutions with finite element models in order to compute the contact forces, taking into account the coupling through gear body deformations.

\subsection{Fixed sun}

Besides the load sharing ratio, the Transmission Error (TE) is an important factor when studying gear transmission behaviour. Although a more detailed analysis of the simulated values obtained with the hybrid model (with analytical and FE features) used in this paper can be shown in [23], a brief highlight of the non-defected transmission error will be shown in this section, to facilitate the appreciation of the effects caused by the configuration and manufacturing and mounting errors. Attending to the transmission 
Table 1: Modeled transmission parameters (mm)

\begin{tabular}{|c|c|c|c|}
\hline & Sun & Planet & Ring \\
\hline Teeth number & 16 & 24 & 65 \\
\hline Module (md) & 4.23 & 4.23 & 4.23 \\
\hline Width & 25 & 25 & 85.9 \\
\hline Pressure angle (tool) & $25^{\circ}$ & $25^{\circ}$ & $25^{\circ}$ \\
\hline Tooth thickness & 6.40 & 8.30 & - \\
\hline Space width & - & - & 8.25 \\
\hline Tip rounding radius & $0.05 \mathrm{md}$ & $0.05 \mathrm{md}$ & $0.05 \mathrm{md}$ \\
\hline Shaft radius & 20 & 20 & 156.4 \\
\hline Number of planets & & 3 & \\
\hline Planets angular phase & & $120^{\circ}$ & \\
\hline Centre's distance & & 86.4 & \\
\hline Elastic modulus & & $207 \mathrm{GPa}$ & \\
\hline Poisson's ratio & & 0.3 & \\
\hline
\end{tabular}

error when the fixed configuration is used, Figure 1 shows how the shape of the resulting error corresponds to the composition of the three independent sun-planet-ring meshes phased out $2 \pi / 3$.

The effect of the transmitted load on the transmission error is threefold: it modifies the average value, the peak-to-peak amplitude and introduces a slight change in the shape of the transmission error curve, as shown in Figure 2 .

The load sharing ratio obtained when a fixed configuration is used represents a direct translation of the relation between the meshing stiffness of each path sun-planet-ring. Thus, in the planetary transmission used in this paper the load sharing among planets undergo strong variations, as shown in Figure 3, reaching differences up to $\pm 9 \%$ of the total load. With respect to the theoretical load capacity of the planet, this difference of $9 \%$ in the total load transmitted entails a $\pm 27 \%$ variation. This means that, although the dynamic behaviour should improve due to the smoother transmission error achieved with the phased-out configuration, the real load transmitted by the gears is about one third higher than the theoretical one.

It could be assumed that this considerable fluctuation in the load transmitted by each path of the planetary transmission should have serious con- 


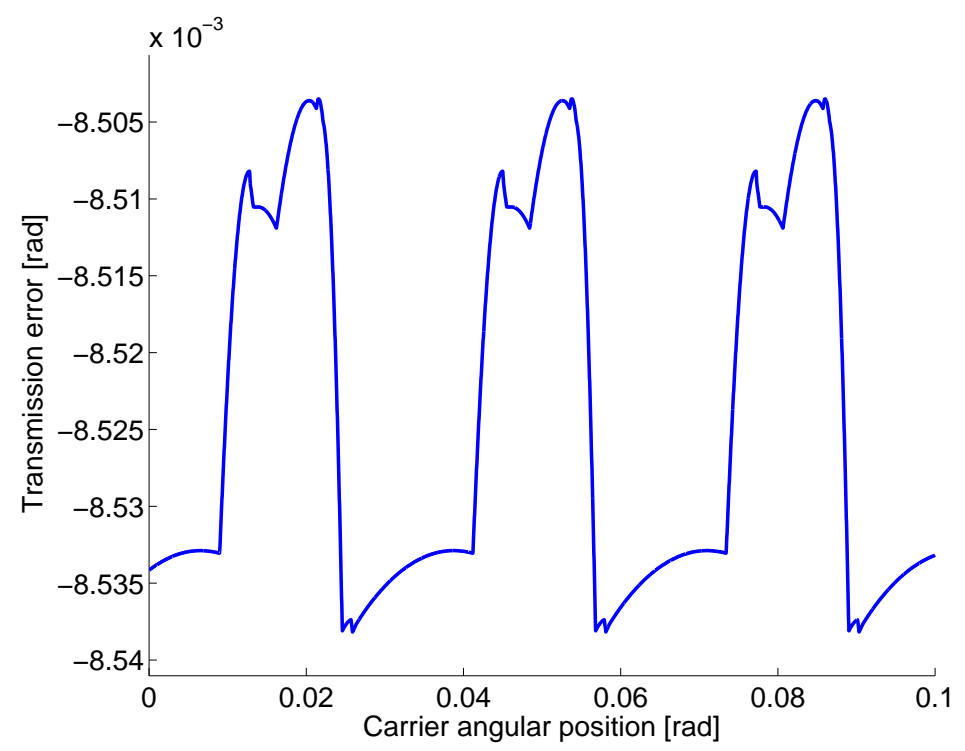

Figure 1: Transmission error with a fixed sun configuration

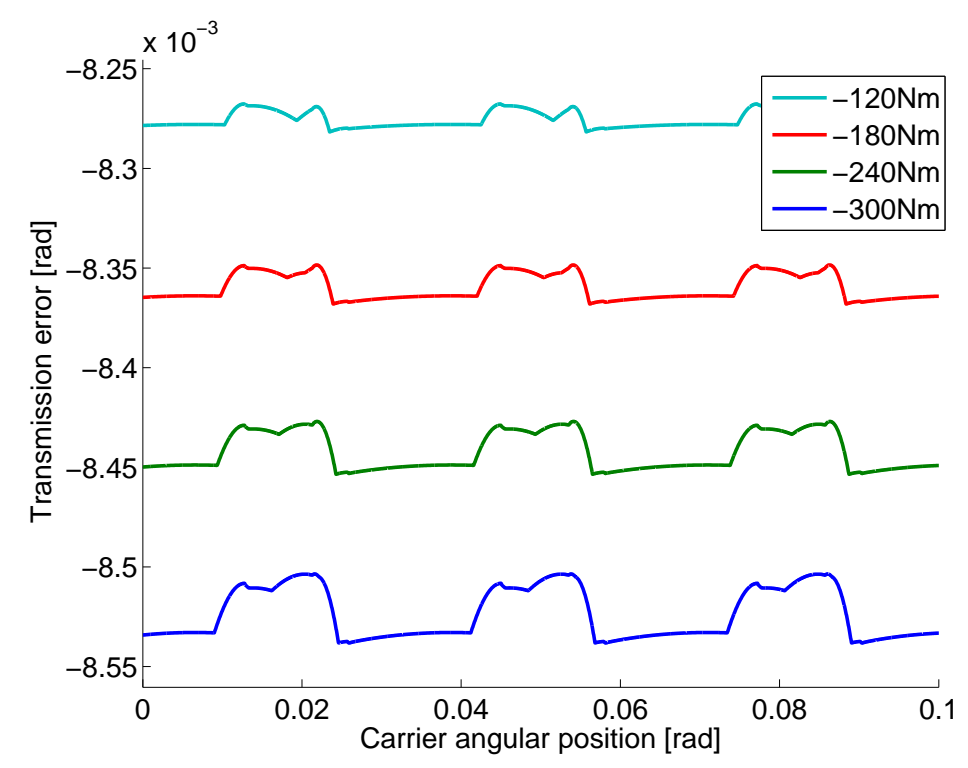

Figure 2: Effect of the load on the transmission error with a fixed sun configuration

sequences on the durability of the gear teeth. However, taking into account the tooth load sharing ratio throughout the meshing cycle shown in Figure 4 , it can be appreciated that although the transmitted torque fluctuates up 


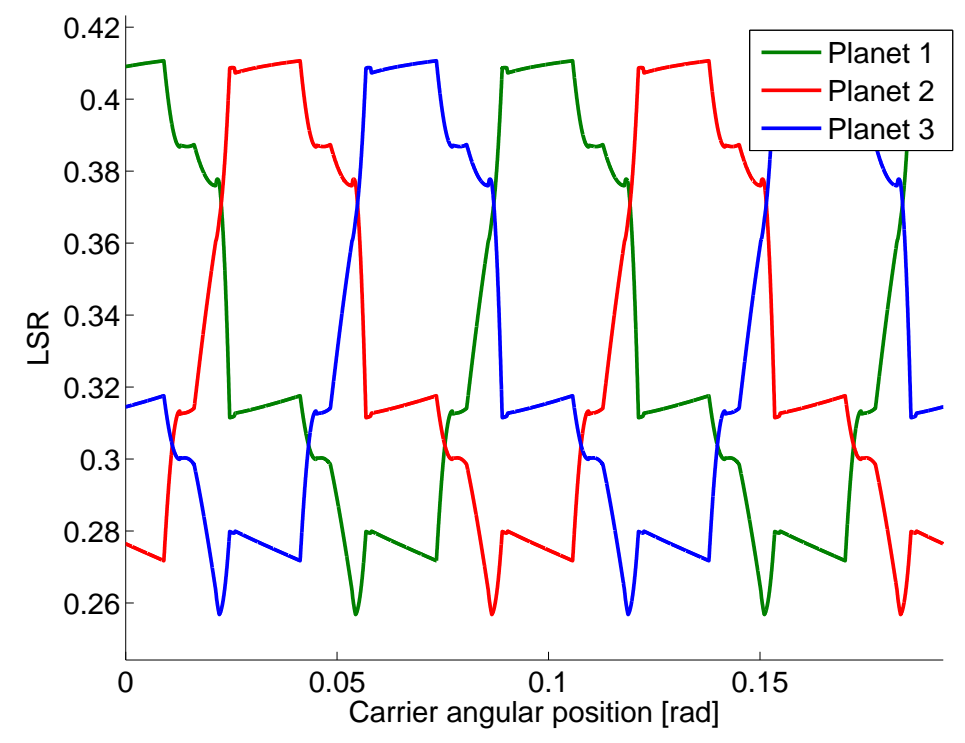

Figure 3: Load sharing ratio with a fixed sun configuration

to a $\pm 27 \%$ variation, the force does not even reach the $100 \%$ of its nominal 198 value.

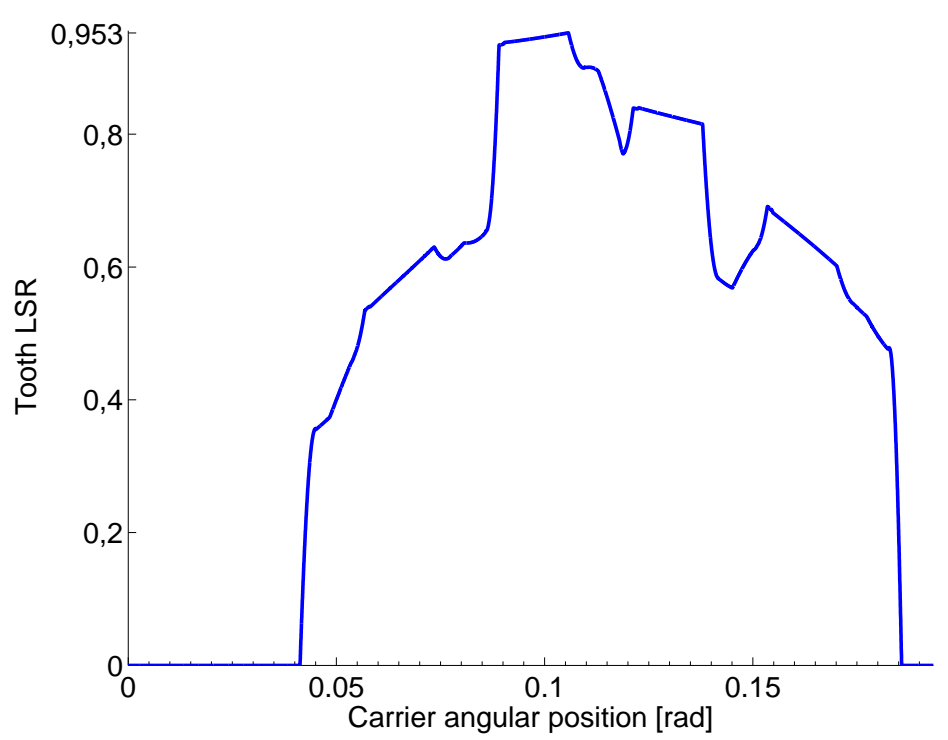

Figure 4: Tooth load sharing ratio with a fixed sun configuration 
To understand this fact, it is necessary to explain how it is calculated the tooth load sharing ratio, which is the ratio force-in-the-teeth to maximumtheoretical-force in the teeth. The numerator is the value of the contact force simulated in the teeth, and the denominator is the theoretical force that equilibrates the transmitted torque when applied at a base-radius distance, divided by the number of paths. In other words, the tooth load sharing ratio represent the difference between the tooth force in the real transmission and the tooth force in a theoretical transmission with only one teeth in contact for each path, transmitting in even load sharing conditions. It is then easy to understand that the fluctuation of $\pm 27 \%$ in the transmitted load by the planet does not necessarily imply the same fluctuation in its teeth. The maximum peak of transmitted load corresponds to the maximum value of the path stiffness, which takes place precisely during the double contact period. Thus, the $+27 \%$ is shared by a teeth pair, and the corresponding tooth load sharing ratio falls below the unit value. This fact can be appreciated comparing Figure 3 and Figure 4, where the tooth load sharing ratio corresponds to the planet 3 planet load sharing ratio both in blue.

\subsection{Floating sun}

When the configuration used includes a floating member, as the sun in this case, the load sharing situation changes drastically. The sun has to meet the static equilibrium of forces in addition to the torque equilibrium, and this causes the sun making an orbit around its central position, as shown in Figure 5. This motion corresponds with the sun keeping away from the most stiff meshes in the direction of the meshing lines, until it reaches a point where the sum of products of the individual overlapping distances and their corresponding stiffness values are equal for all paths.

In Figure 6, three graphic constructions are shown to help visualize the motion of the sun when it is allowed to move freely. At the top sub-figure, it is represented the forces and torque equilibrium situation for the fixed sun configuration. The system of gear forces results in a balance of torques with a nonzero force component caused by the greater stiffness of the planet-sun mesh 1 (in red).

When the sun is released from all constraints, it will translate and rotate to find the new position of equilibrium. For the shake of clarity, the movement is decomposed in two independent parts: translation and rotation.

Starting from the initial graphic construction, the sun must move away from the most rigid contact (mesh 1), until the forces triangle becomes closed 


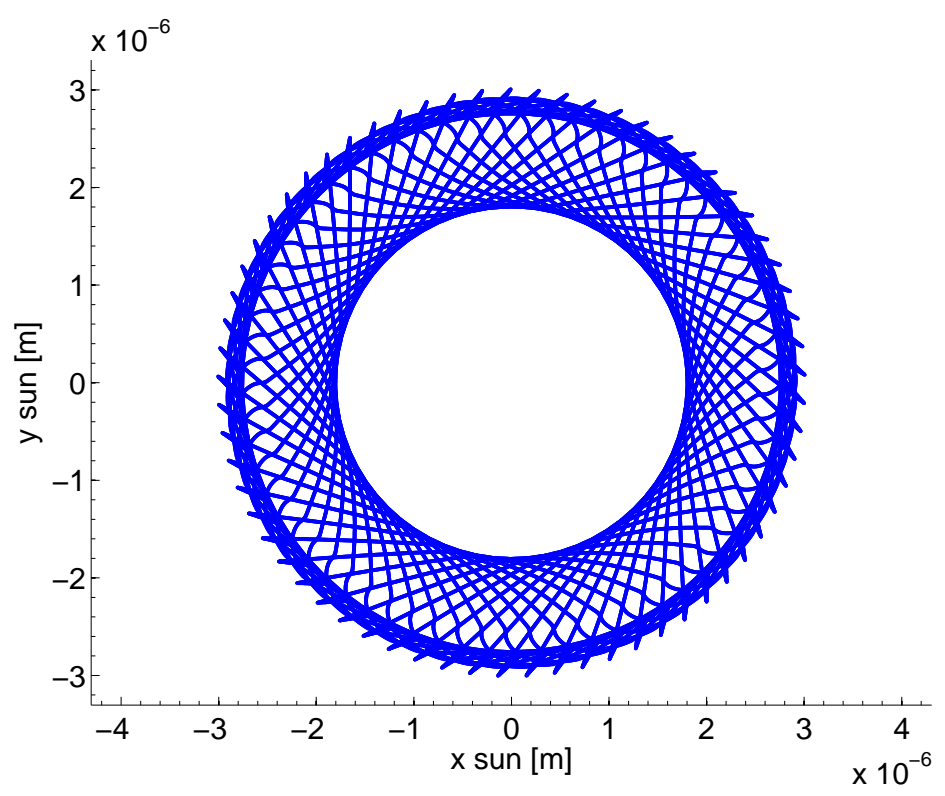

Figure 5: Sun orbit with a floating sun configuration

(equilateral due to the symmetry of the problem). The gear contact overlap of mesh 1 is reduced by a length of $\Delta \delta_{1}$. Consequently, the other two gear contact overlaps will increase by certain lengths $\Delta \delta_{2}$ and $\Delta \delta_{3}$. These variations in the overlap distances will have proportional consequences in the values of the contact forces. There will be a position of the gear center that will comply with the equilibrium of forces, closing the triangle as shown in the second sub-figure.

Since the base radius remains unchanged during these operations, the torque values due to the contact forces will be consequently proportional to said contact forces. In the position of the gear center found in the first movement of the sun, the equilibrium of forces has been satisfied, but now the balance of torques has varied, and the torques produced by the gear meshes are not balanced with the external applied torque $T_{\text {ext }}$. As the position of the gear center guarantees the force equilibrium, to balance the external torque will suffice with the addition of a certain rotation to the gear, increasing the overlaps and thus the sum of the individual torques. These two steps should be repeated until both equilibrium conditions are satisfied: translations to maintain the forces triangle closed (equilibrium of forces) and rotation to maintain the torque equilibrium. 

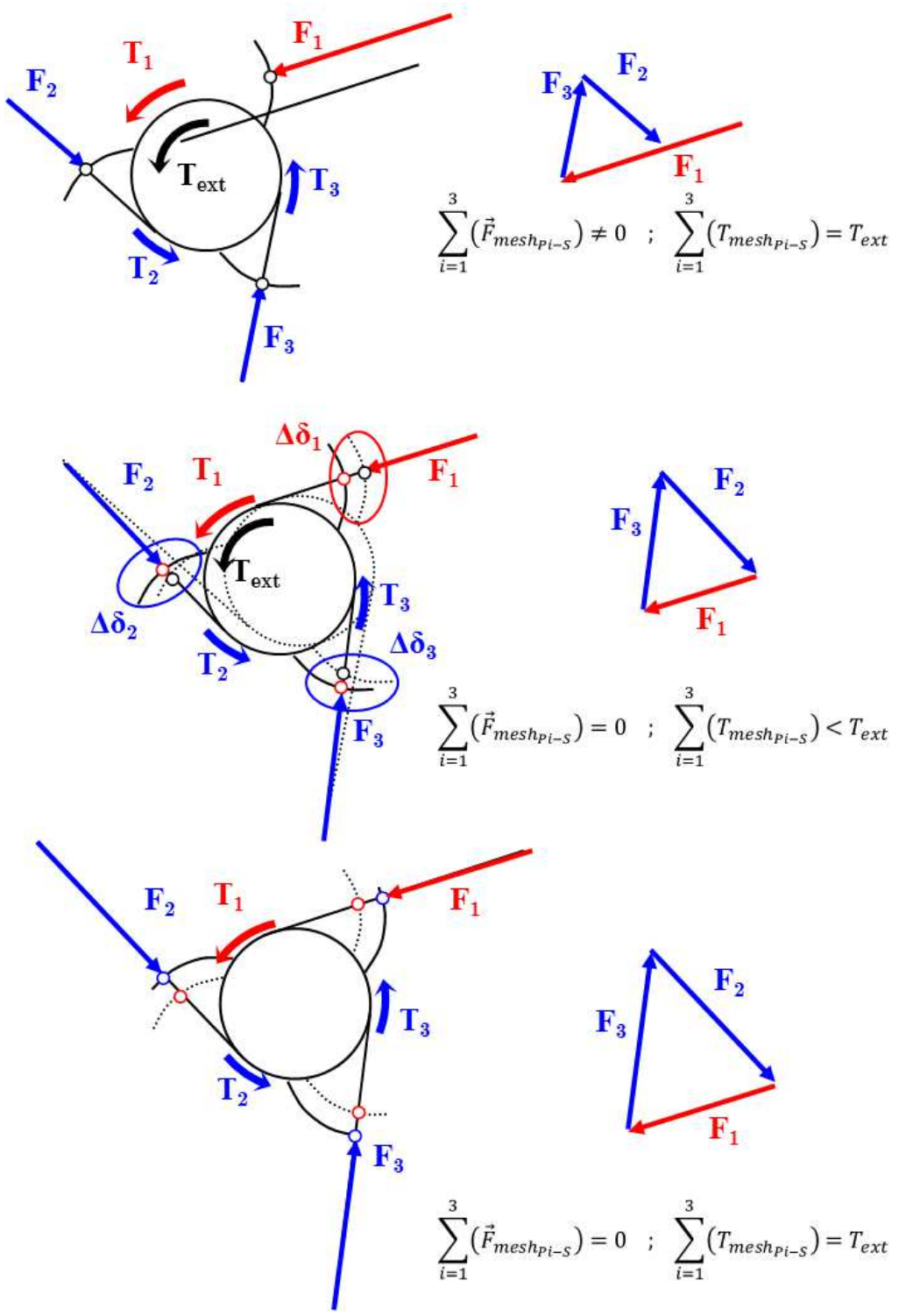

Figure 6: Translation and rotation of the sun due to variable meshing stiffness

Understanding this necessary composition of movements for the sun when it is released from its bearing constraints can also help to understand two important consequences in the transmission behaviour. First of all, due to the necessity of satisfy the forces equilibrium and the symmetry of the problem, the load sharing must be even among planets. Each planet will carry 
the same force vector (one side of the equilateral triangle shown in the Figure 6). The second consequence is related to the transmission error signal obtained from the planetary transmission, shown in Figure 7. The gear has to perform an extra rotation with respect to the configuration with fixed center, to compensate the loss of torque due to the translation. Another interpretation of this fact, with maybe more profound implications, is that the implementation of floating central members in planetary transmissions brings, as a consequence, the reduction of the overall rotational stiffness of the system.

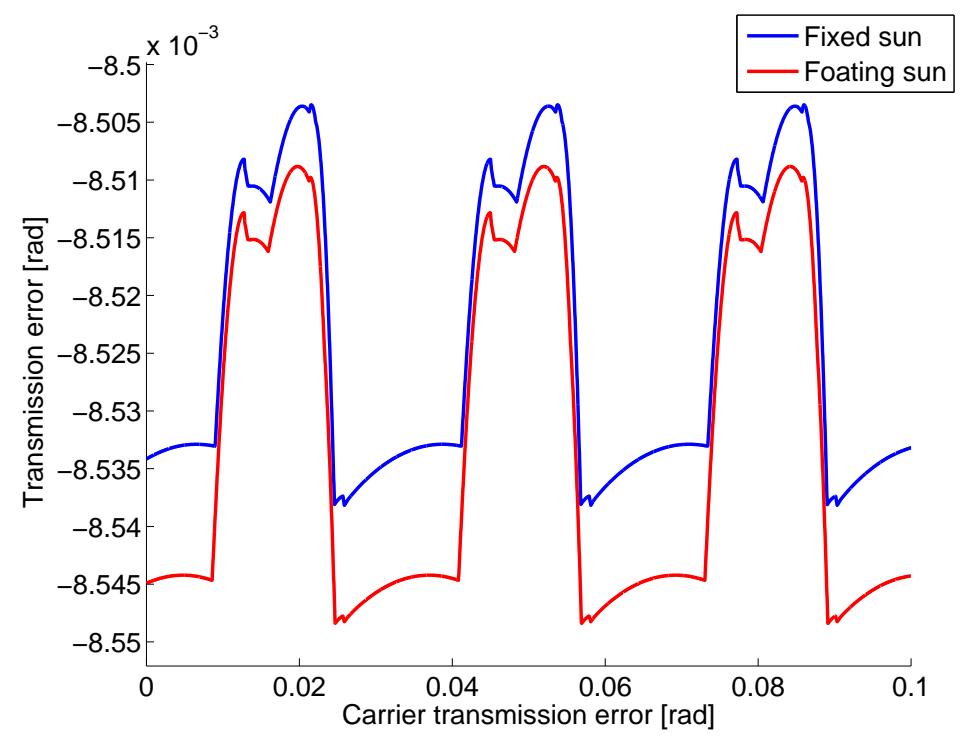

Figure 7: Transmission error with a fixed sun vs. floating sun

As stated, the LSR must be even for all the load paths, as shown in Figure 8. There are some negligible differences in the distribution, due to the loss of symmetry when contacts out of the line of action take place (the example transmission does not present profile modifications, and consequently there are corner contacts in the teeth tips).

\section{Errors in planetary transmissions}

It is easy to find numerous references in the literature dealing with the behavior of planetary train in the presence of defects, especially after the recent boom in transmissions computational modeling. 


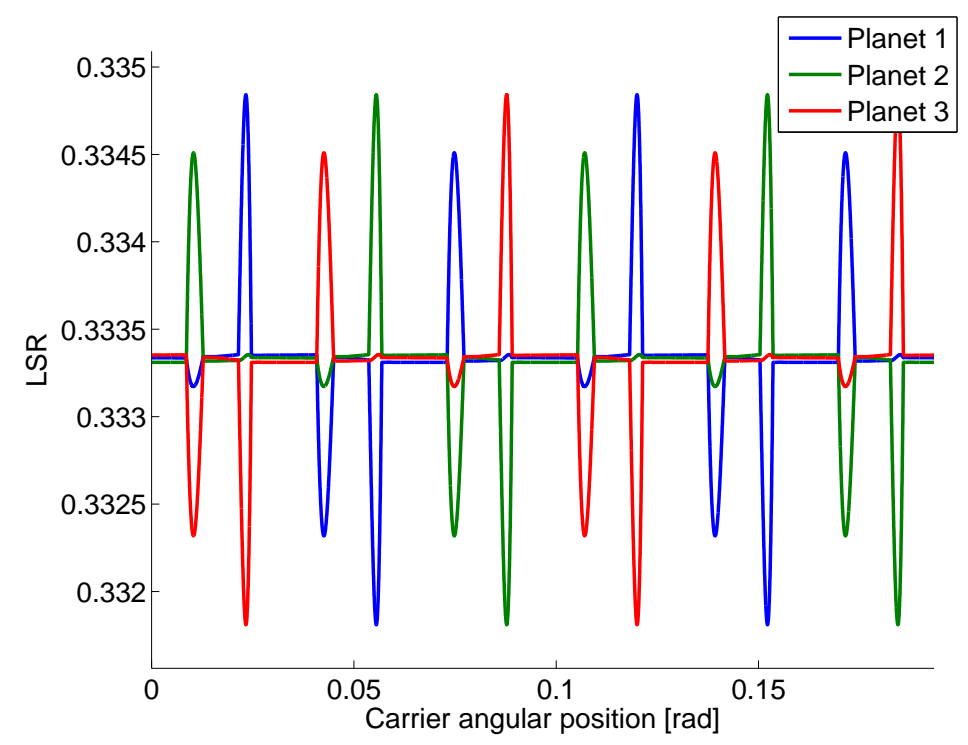

Figure 8: Load sharing ratio with a floating sun configuration

A large percentage of the published works are based on the study of the Load Sharing Ratio, analyzing the causes and defects for which an even distribution does not occur, and proposing solutions or tools for predicting the amount of imbalance [26, 27]. It is generally accepted that transmissions with 3 planets are those with a better load sharing capability. The option of configuring the transmission with a floating central member is the solution that provides the best results, absorbing deviations, manufacturing and assembly errors, and improving the LSR.

There are two sources related to manufacturing and assembly errors which are known to have a strong impact on the load sharing distribution in planetary transmissions: the errors in the positioning of the planets [26] and the eccentricity of the wheels [28]. The following describes each of the errors and their implementation on the model developed.

\subsection{Planet pinhole positioning errors}

The planet positioning error occurs when due to manufacturing tolerances of the carrier, the planet shaft positions differ from their theoretical location. As the impact of this error is determined by its direction, in this study the error will be divided in two components: radial or tangential component, as shown in Figure 9. For simplicity and clarity, the positioning error effect will 
be studied in one single planet, remaining the other two planets located in their nominal position. As the considered errors are sufficiently small, the variation of the pressure angle will be neglected.

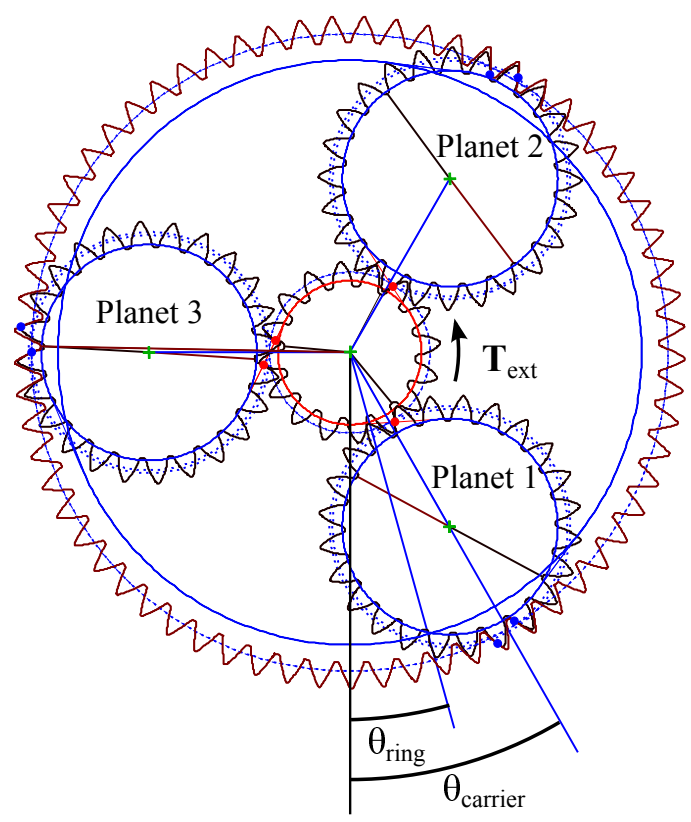

Figure 9: Planet position errors

The positioning error of the planet is decomposed into the $e_{\text {tan }}$, parallel to the line tangent to the circumference at the planet center points, and the $e_{\text {rad }}$, which corresponds to deviations in the radial direction towards the center of the system. The sign of the value is positive when the deviations coincides with the direction shown in Figure 9.

The tangential component of the positioning error has the effect of advancing or delaying the beginning of the contact teeth of the affected planet, depending on the direction of the error. In Figure 10 it is shown a planet with positive error $e_{t a n}$. It can be appreciated that the movement of the planet centre with respect to its theoretical position causes the apparition of variation in the geometric overlap for the sun-planet $\left(\Delta \delta_{S-P}\right)$ and ringplanet $\left(\Delta \delta_{P-R}\right)$ meshes respectively. These variations in the geometric overlaps bring the consequent variation of the associated contact forces in both meshes, and thus in the torque transmitted by the corresponding sun-planetring path affected with the error. In this case, the positive error combined 

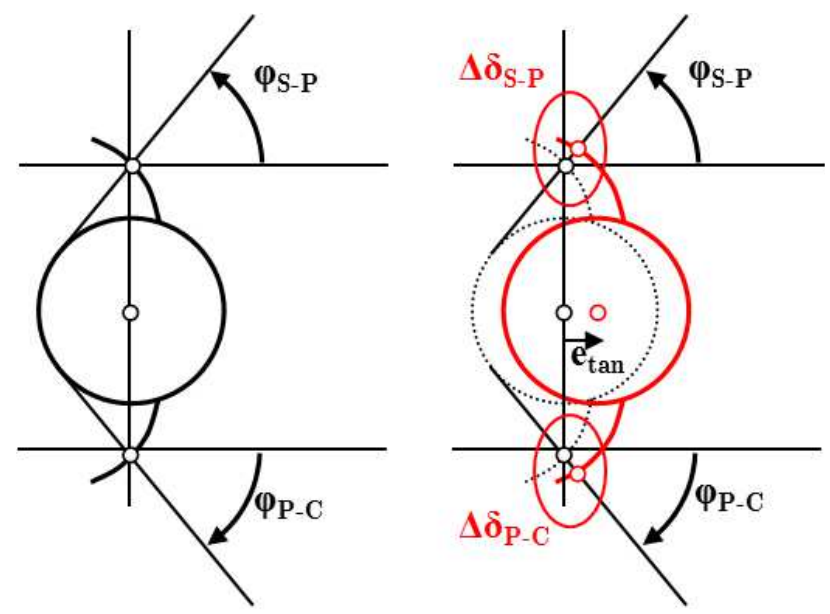

Figure 10: Overlapping variation due to $e_{t a n}$

with the direction of the torque, results in a preload of the affected planet. This excess of transmitted torque $\Delta T$ is given by:

$$
\begin{gathered}
\frac{\left(k_{P-R} \Delta \delta_{P-R}+k_{S-P} \Delta \delta_{S-P}\right)}{2}=\Delta T \\
\text { where }\left\{\begin{array}{l}
\Delta \delta_{S-P}=e_{\text {tan }} \cos \left(\varphi_{S-P}\right) \\
\Delta \delta_{P-R}=e_{\text {tan }} \cos \left(\varphi_{P-R}\right)
\end{array}\right.
\end{gathered}
$$

Where it can be appreciated the influence of the contact stiffness $k_{S-P}$ and $k_{P-R}$ and the pressure angles $\varphi_{S-P}$ and $\varphi_{P-R}$.

The amount of excess transmitted torque is much lesser for radial positioning errors depicted in Figure 11.

In this situation, the displacement of the planet produces a variation in the geometric overlapping $\Delta \delta$ in the opposite direction for each mess. Thus, while the overlap variation is positive in the case of the sun-planet mesh, it is negative for the planet-ring mesh. As the torque balance in the planet must be zero, it seems apparent that the planet will suffer a rotation moving away from the sun-planet contact and closing the gap opened in the planetring contact. If the geometric overlaps are the same in absolute value, they cancel each other and the only effect of the planet radial displacement is an additional rotation of the gear with consequences in the phase of the system, but no effect on the transmitted load. Nevertheless, the fact that in some planetary drives the pressure angle of the sun-planet pair is different than the planet-ring has been ignored by some authors. It is common to find in 

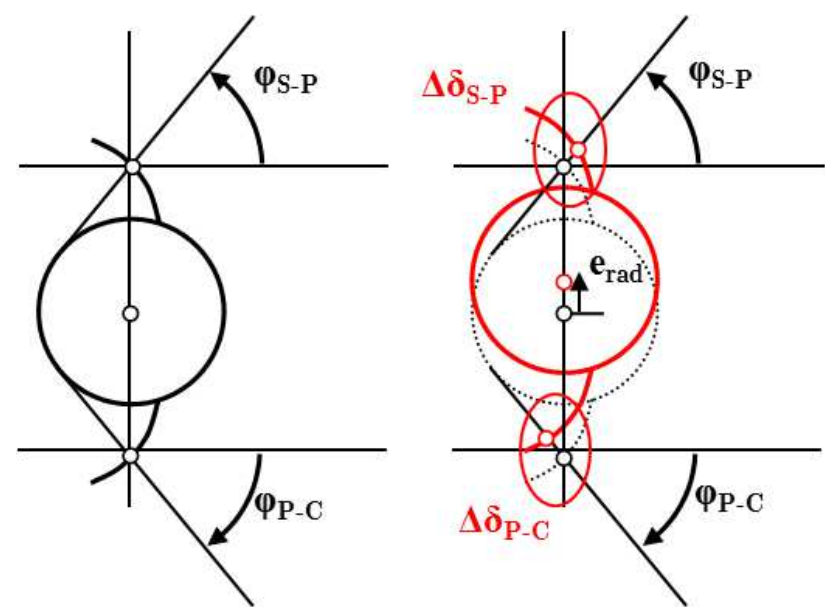

Figure 11: Overlapping variation due to $e_{\text {rad }}$

the literature assertions about the negligible effect of the radial component of the positioning error on the load distribution through the different paths in planetary transmissions [26].

Whilst it is true that the compensatory nature of the overlaps produced by the radial movement of the planet minimizes its effect on the load sharing ratio (especially when compared to the case of the tangential displacement), the LSR is far from perfect when there are differences in the pressure angles, as in this case:

$$
\begin{aligned}
\Delta \delta_{S-P} & =e_{\text {tan }} \sin \left(\varphi_{S-P}\right) \\
\Delta \delta_{P-R} & =e_{\text {tan }} \sin \left(\varphi_{P-R}\right)
\end{aligned}
$$

\subsection{Run-out errors}

Eccentricity (run out) occurs when there is a difference between the geometric centre of the gear and the position of its rotation centre. Analyzing its nature, from a kinematic point of view, the run-out error is a kind of positioning error in which the radial and tangential components vary sinusoidally with the angular position of the planet gear (or carrier angular position). Thus, the implementation of eccentricity is based on the positioning error implementation, with the added complexity of the sinusoidal variation function of the planet gear centre angular position. 


\section{Modeled behavior of the planetary transmission with errors}

As stated before, the fewer degrees of freedom, the greater impact of errors in a transmission has. As compliances are introduced, the load sharing behavior improves, as it has been demonstrated by means of theoretical, geometric and modelling approaches in the previous sections. In the following, positioning errors will be analyzed by components in order to ratify the conclusions drawn from geometric analysis presented before. To illustrate the effect of the configuration on the LSR, the results obtained for a fixed sun will be shown first, and afterwards the improvement of the behavior when the sun is allowed to orbit will be presented.

\subsection{Tangential component of the positioning error in fixed sun configuration}

In Figure 12, it is shown the LSR for a planetary transmission with fixed sun and an $e_{\tan }$ of varying magnitude in planet 1 . In a dashed line, at the center of the figure, it is reproduced the LSR for a transmission without defects (same results as those shown in Figure 4) to be used as reference. In a dotted line, the results of LSR for a tangential positioning error of $30 \mu \mathrm{m}$ are shown. At this level of error the planet 1 assumes at certain positions of the meshing cycle up to $80 \%$ of the total transmitted load $(900 \mathrm{Nm}$ at the sun).

The loss of symmetry in the results is generalized. The non-faulty planets present of course a very different level of load, but also the shape and amplitude of the LSR curve is affected, now having three different forms of LSR for each planet. When the positioning error is increased to $50 \mu \mathrm{m}$, the overlap variation of the planet 1 is such to cause the complete unloading of the non-faulty path loads, and the defected planet carries the entire amount of transmitted torque.

The effect of a tangential error in the opposite direction (negative error) is contrary to the previous results shown. Instead of increasing the overlap distance between profiles in the planet, this error spread the profiles apart, avoiding contact and thus the transmission of load. This fact can be confirmed by the LSR results shown in Figure 13, where there can be appreciated a drop of the load carried by planet 1 when a tangential negative error of 20 $\mu m$ is introduced.

Another conclusion can be drawn from the different effect that positive or negative tangential error have on the LSR behaviour. When a planet is affected by a tangential error, in an extreme case of a negative error, the 


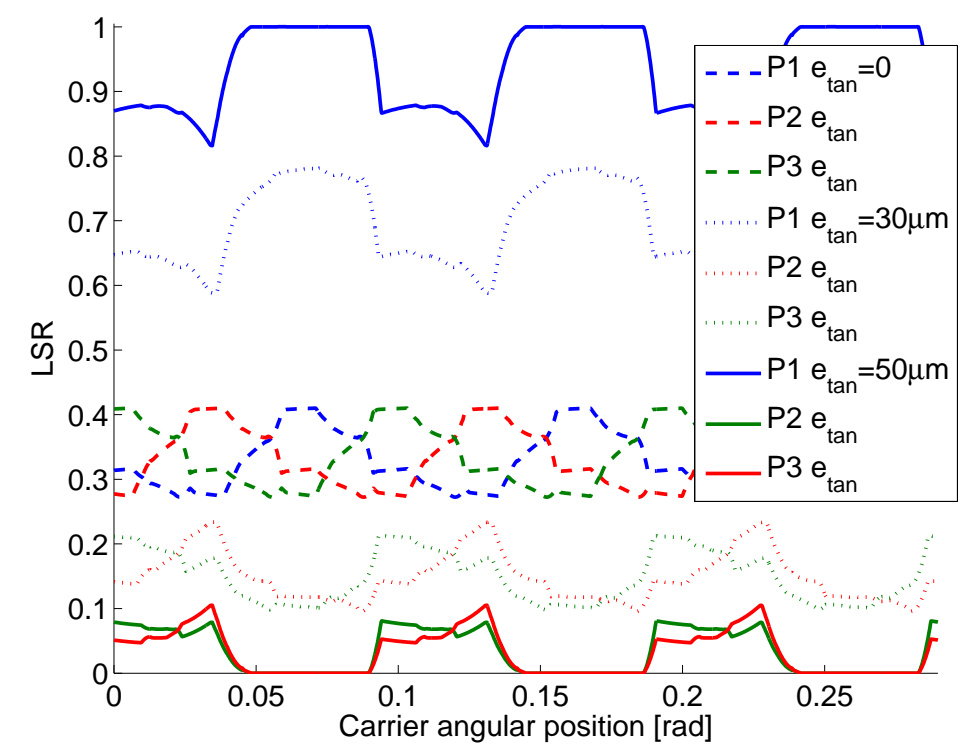

Figure 12: Load sharing ratio with fixed sun and positive $e_{t a n}$

faulty planet becomes unloaded and the rest of the paths shares the excess of torque. Nevertheless, for the extreme case of positive error, the faulty planet assumes the whole amount of load. It is apparent that it is far more critical a situation in which a single planet is loaded with all the transmitted torque (positive error) than the situation in which a set of planets must share the load corresponding to the faulty unloaded planet (negative error).

To summarize the effect of the tangential error in the LSR, in Figure 14 the LSR of planet 1 is shown for a range of $e_{t a n}$, from negative to positive values. While the shape of the curves along the cycle varies strongly with the error magnitude, the average level of the LSR varies proportionally with the positioning error.

As it was first postulated in section 3.1, it could be assumed that these considerable fluctuations in the load transmitted by each planet of the transmission should have proportional impact on the teeth forces, with consequences on the gears durability. Looking again at the tooth load sharing ratio of planet 1 throughout the meshing cycle shown in Figure 15 for an $e_{\text {tan }}$ of $30 \mu \mathrm{m}$. Now, attending to Figure 12, planet 1 carries roughly $60 \%$ to $80 \%$ of the total transmitted load. Since the nominal load assigned to a planet in a 3 planet transmission is $1 / 3$, this means that planet 1 is transmitting between 1.8 and 2.4 times the assigned value of the load. Nevertheless, in 


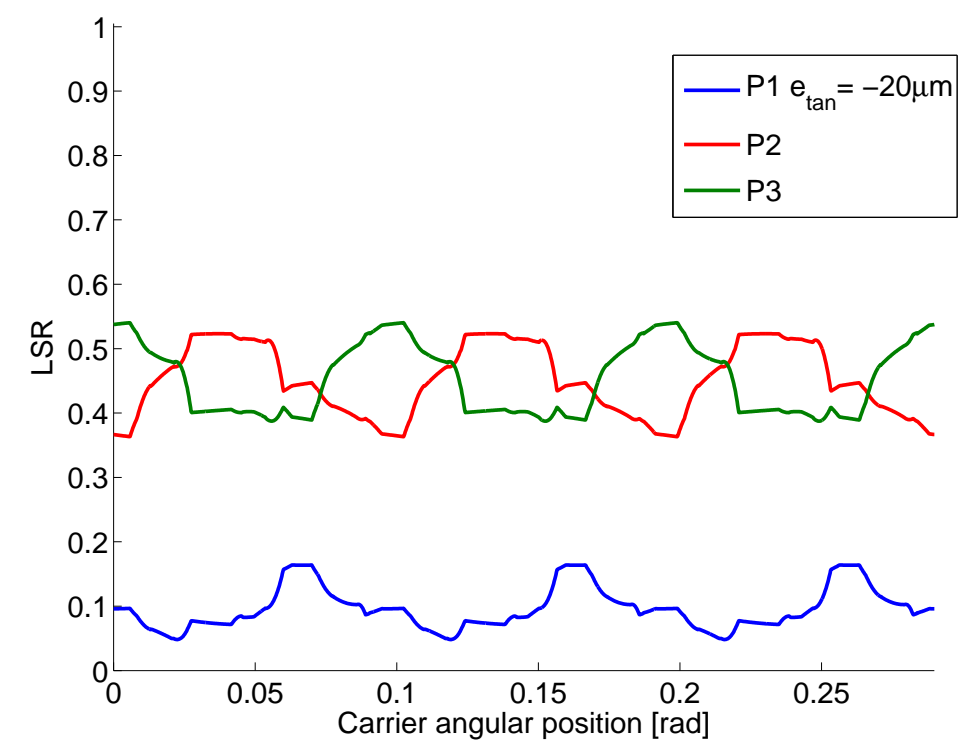

Figure 13: Load sharing ratio with fixed sun and negative $e_{t a n}$

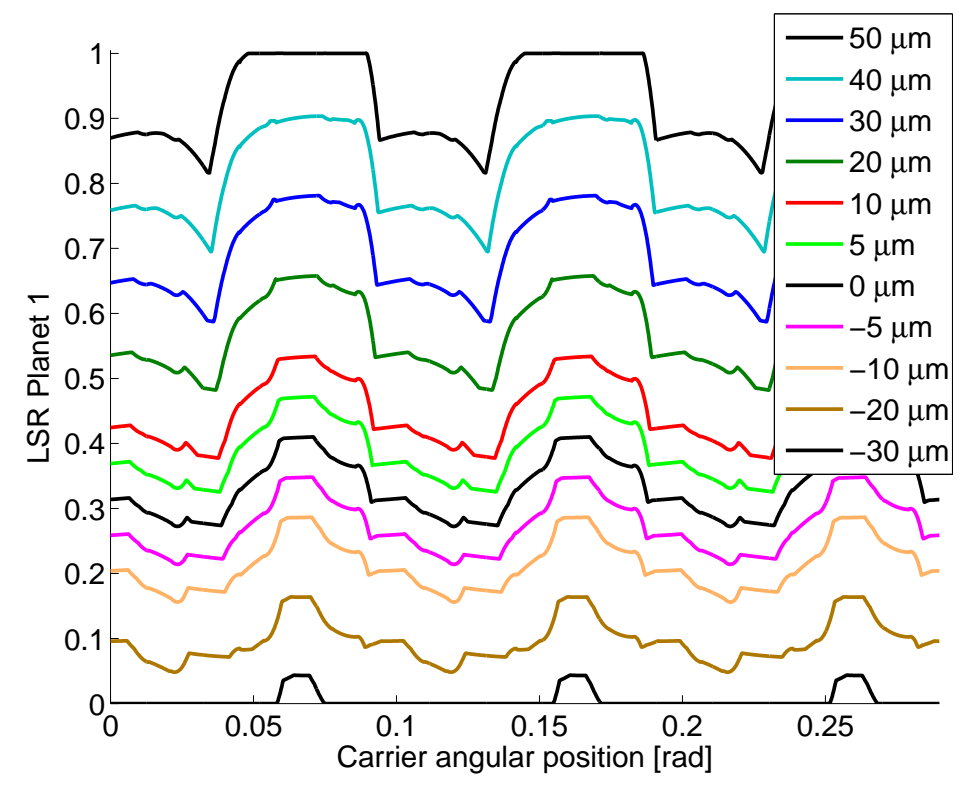

Figure 14: Load sharing ratio with fixed sun and positive and negative $e_{t a n}$ 
contact presents, allowing the rest of the paths with more teeth pairs in mesh to carry corresponding greater forces.

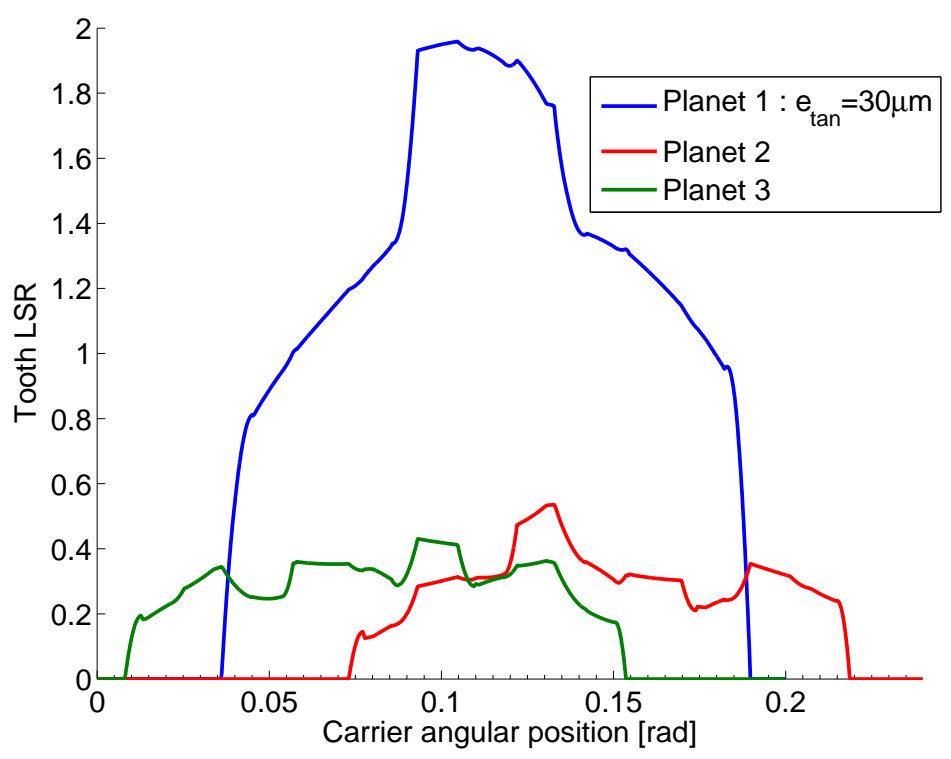

Figure 15: Tooth load sharing ratio with a fixed sun configuration and $e_{t a n}=30 \mu m$

The effect of the torque level on the LSR is very relevant, as shown in Figure 16. In general, geometry related errors in gear transmissions have a higher visibility for lower load levels, as higher loads imply greater elastic deformations and therefore the dilution of the error deflections.

Although the focus of this study is centered on the LSR consequences of manufacturing errors, it is evident that there are other measurable signals that show disturbances due to these errors. The transmission error presented in Figure 2 has three lobes per planetary meshing period. In Figure 17 the same planetary transmission error is shown (for an input torque of 900Nm), when different and progressively greater values of $e_{\tan }$ are introduced in planet 1. As the faulty planet gradually assumes the torque corresponding to the other two loading paths (planets 2 and 3), the transmission error lobes caused by these unloading planets become reduced and absorbed by the shape of a transmission error characteristic of a sun-planet-ring transmission.

\subsection{Radial component of the positioning error in fixed sun configuration}

The radial component positioning error of the planets, as introduced in Section 4, has a much lesser effect on the LSR than the tangential compo- 


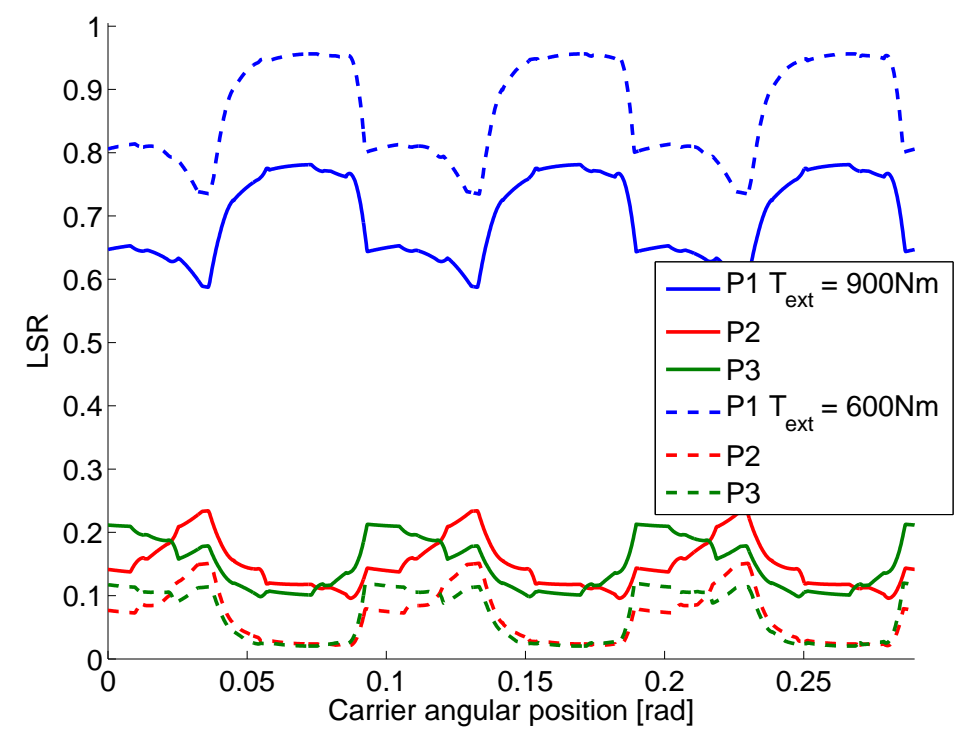

Figure 16: Load sharing ratio with a fixed sun configuration and $e_{t a n}=30 \mu \mathrm{m}$ and different loads levels

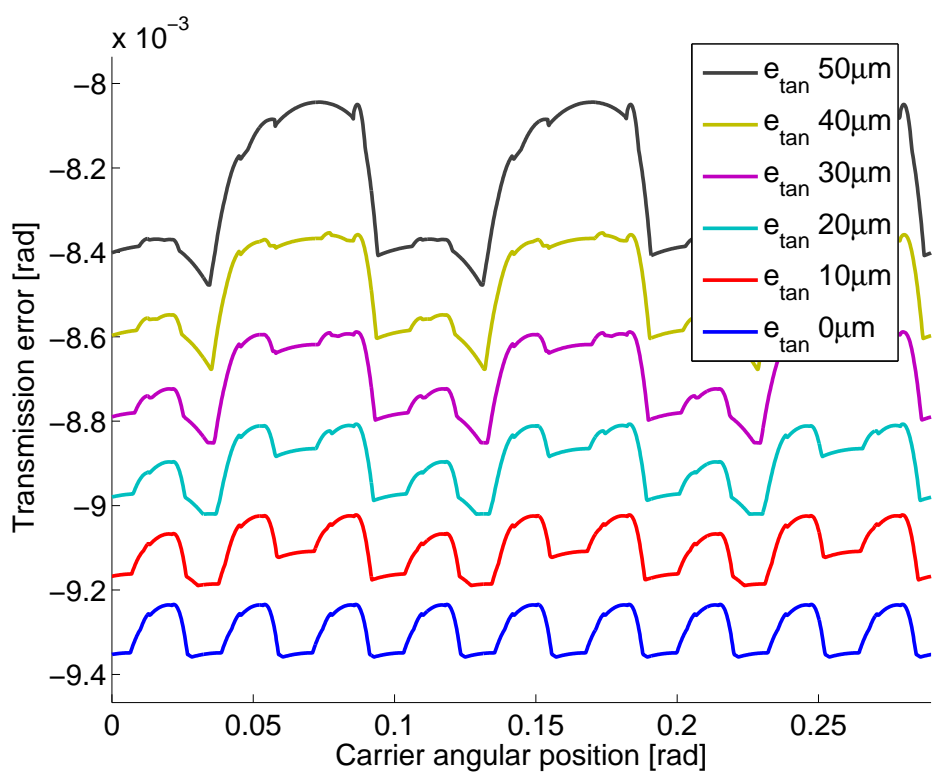

Figure 17: Transmission error with a fixed sun configuration under different $e_{\text {tan }}$ values

nent. It mainly depends on the difference between the operating pressure 
angles of the sun-planet and planet-ring meshes, and its effect is practically nonexistent when these angles are equal (which is usual). In the example transmission, the operating pressure angles for each gear pair are calculated for the planetary transmission as $\varphi_{P-R}=24.45^{\circ}, \varphi_{\mathrm{S}-\mathrm{P}}=27.37^{\circ}$.

Figure 18 shows the LSR of the transmission when a radial positioning error of $40 \mu \mathrm{m}$ is introduced in planet 1 . The difference between the average LSR value of the faulty path track relative to the other two is about 2 percentage points, which shows the much lesser impact of the radial component compared to the results shown in Figure 12.

In order to illustrate the comparison between both component effects of the positioning error, it has been found that an error of $40 \mu \mathrm{m}$ in the radial direction is equivalent to a tangential error of $1 \mu \mathrm{m}$ in terms of LSR impact. Both cases are shown in an overlapping manner in the Figure to assess its coincidence. The LSR values corresponding to the tangential error are shown in black and different types of dashed line.

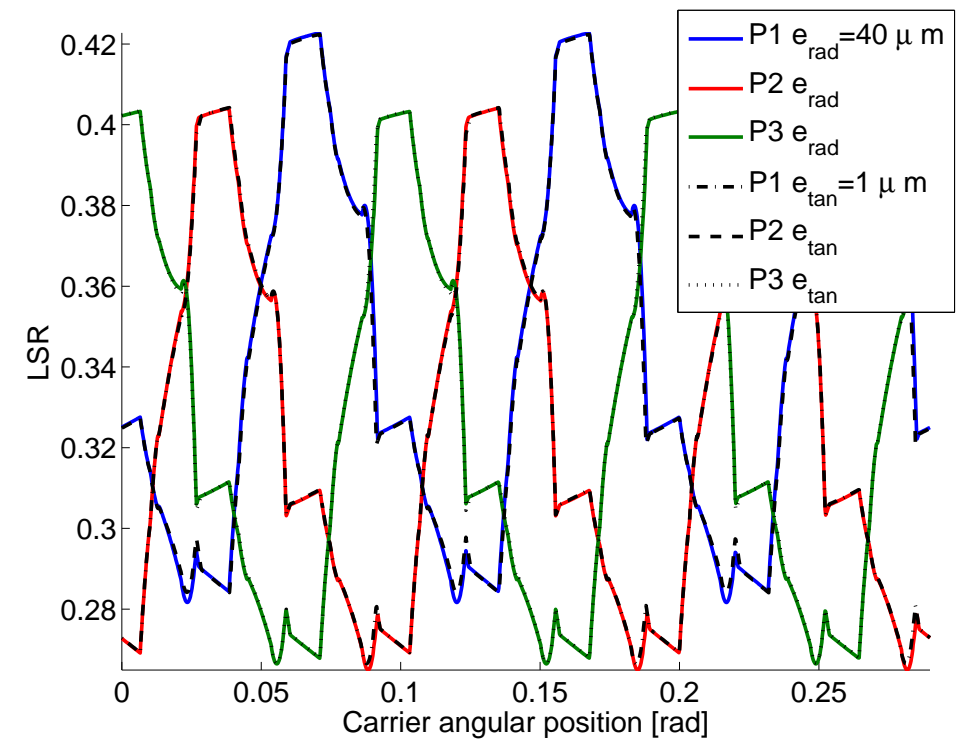

Figure 18: Load sharing ratio comparison with fixed sun between $e_{\text {rad }}$ and $e_{\text {tan }}$

\subsection{Positioning errors in floating sun configuration}

When the configuration of a planetary transmission with positioning errors is modified from fixed to floating sun, the LSR becomes homogenized approaching a perfect $1 / 3$ for each load path (or planet). The sun describes 
an orbit around its central position, compensating for the positioning error of the planet by adjusting its separation distance (and thus overlap distance) in the direction of the line of action, as shown in Figure 19.

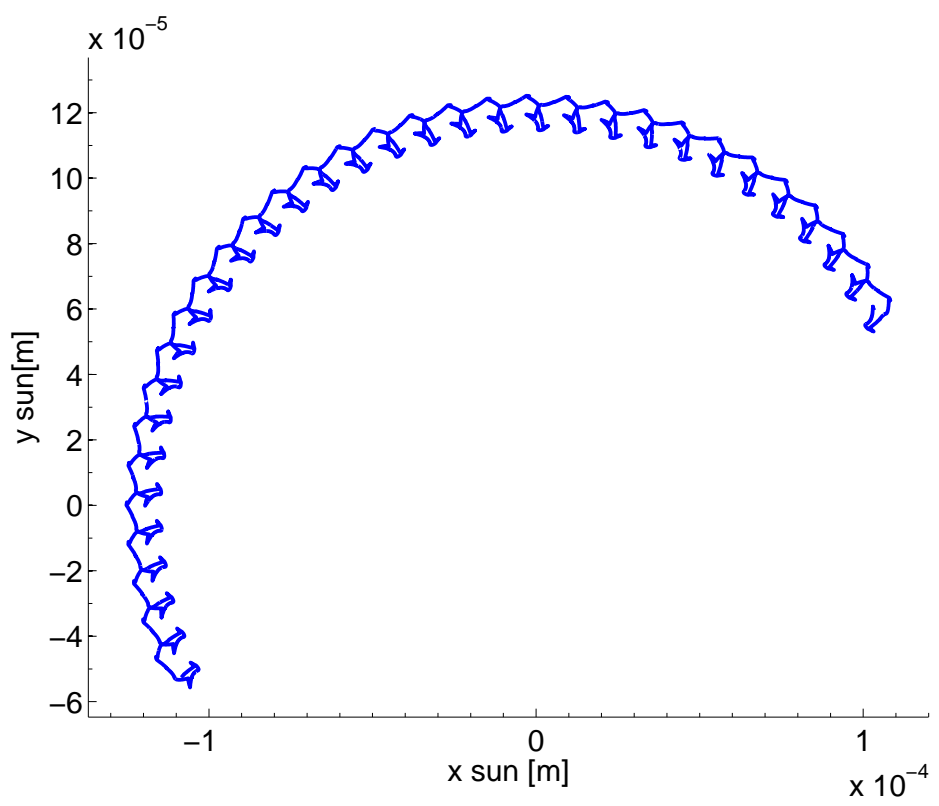

Figure 19: Sun orbit with $e_{\text {tan }}=30 \mu m$

In this figure, the sun orbit is shown for positions of the carrier from 0 to $\pi$ radians, and considering a positive tangential positioning error of planet 1 of $100 \mu \mathrm{m}$. For the initial position shown in the figure, it can be seen how the sun moves away from the center in the same direction as the pressure angle, therefore moving away from the contact with planet 1 . It can be appreciated from the orbit graph that the radius of the orbit described approximates the magnitude of the planet positioning error.

As it was stated before, by describing this orbit, the planetary forms a zero forces system, for which the resulting contact forces must be equal due to the symmetry, arriving as consequence to the final 1/3 LSR shown in Figure 20. The only exceptions for this perfect $1 / 3$ can be found at the contacts out of the line of action at the teeth tip (the peaks in the figure), where the symmetry is broken because of the change in the effective pressure angles. The net differences between the LSR of each planet, which can be appreciated in Figure 20, are also due to a slight and negligible loss of symmetry caused by the $100 \mu \mathrm{m}$ of variation in the vertex of the triangle formed by the three 
planet pin points.

Complementary to the homogenization that occurs in the LSR when the sun is allowed to move, the individual tooth load sharing ratio also regains symmetry for the three planets, as seen in Figure 21. In this case, the maximum value is the unit corresponding to the force that balances a third outer torque applied during simple contact area, as the floating sun now can also compensate for stiffness differences, which did not happened in the fixed sun case shown in Figure 4.

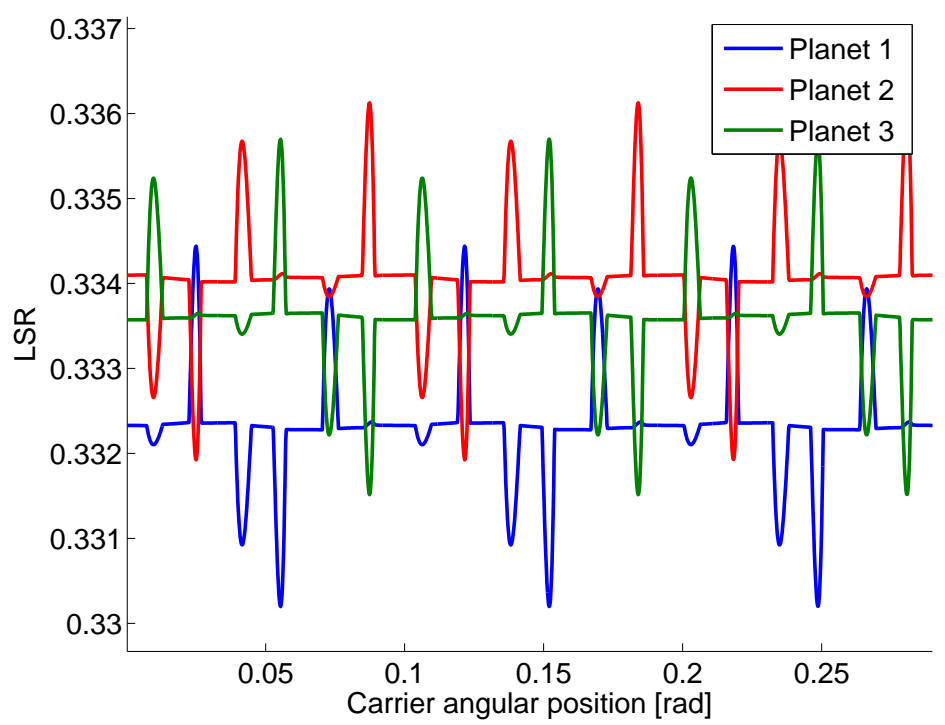

Figure 20: Load sharing ratio with floating sun and $e_{t a n}=100 \mu m$

Regarding the transmission error, the differences between both configurations can be appreciated in Figure 22. As happened in the results without positioning errors shown in Figure 7, the sun must perform an extra rotation to compensate the loss of torque due to orbit translation, for which the transmission error increases. For the floating configuration, the shape of the transmission error exhibits symmetrical behaviour for each path, with three lobes per meshing period, whereas for the fixed sun configuration the predominant faulty planet dominates the transmission error shape.

\subsection{Run-out errors in fixed sun configuration}

All eccentricity simulations presented below are carried out with an error of $20 \mu \mathrm{m}$ in the positioning of the axis of rotation of planet 1 with respect 


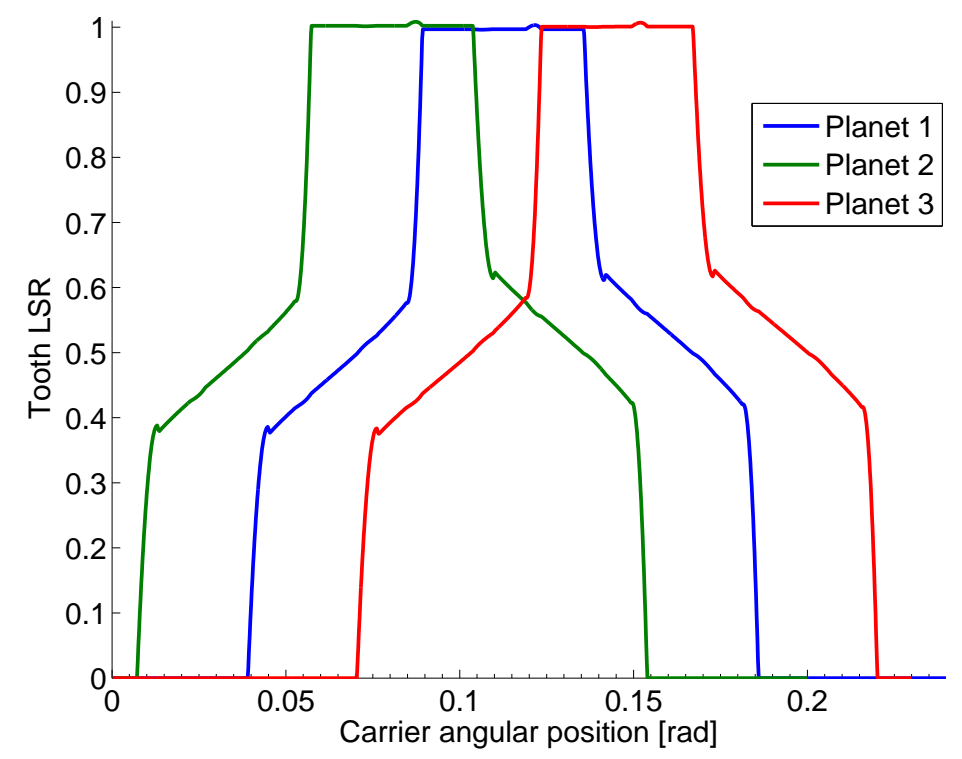

Figure 21: Tooth load sharing ratio with floating sun and $e_{t a n}=100 \mu m$

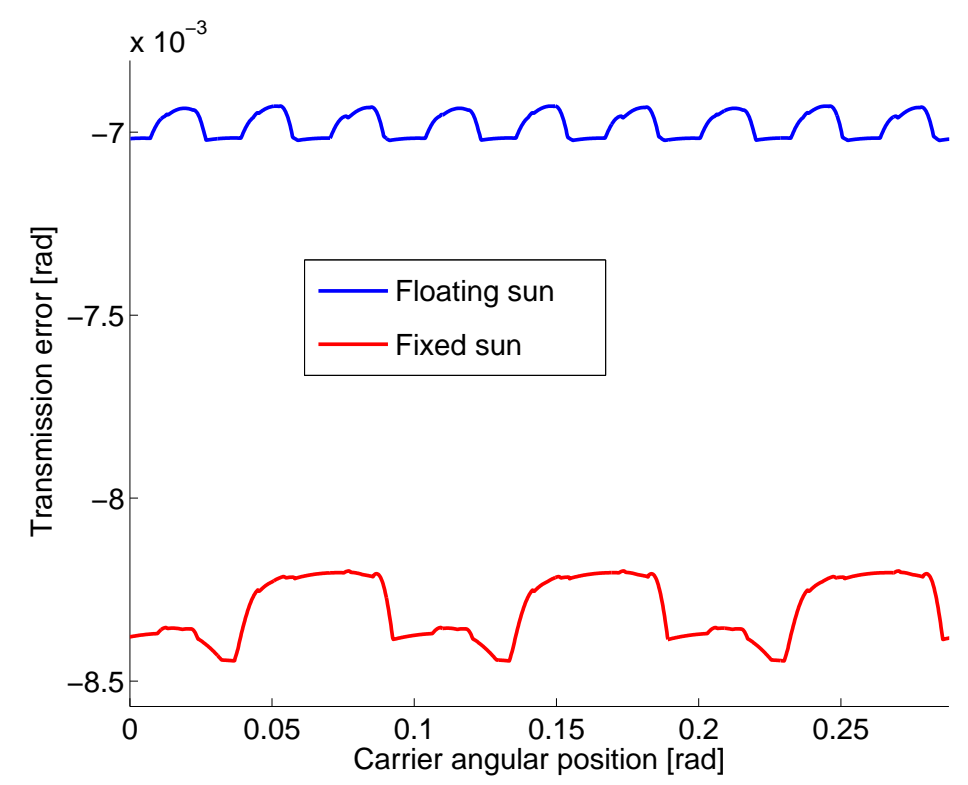

Figure 22: Transmission error with a fixed sun vs. floating sun and $e_{\text {tan }}=30 \mu \mathrm{m}$

to its geometrical center. For the initial position (zero angular position of the planet carrier), the geometric center of the wheel is displaced $20 \mu \mathrm{m}$ in 
the negative radial direction and thus, in this initial position, the planetary configuration coincides to that of a negative $e_{\mathrm{rad}}$ of $20 \mu \mathrm{m}$. Therefore, the LSR behaviour with eccentricity has to be identical as that presented for the transmission with radial positioning error of the same magnitude. Indeed, in Figure 23 it is shown that the LSR curves present very little deviation from the $1 / 3$ load sharing that would be expected with an error of radial positioning of such relative low magnitude. As the planet carrier rotates counterclockwise, the planet does clockwise, and the projection of the eccentricity of each of its components begins acquiring a negative tangential component, which progressively causes the unload of the planet as seen in the previous sections. As the system rotates, the tangential component increases and the radial decreases, up to the point where the planet performs a relative rotation of $\frac{\pi}{2}$ with respect to the carrier. At this point, the eccentricity will place the geometric center of the planet at $20 \mu \mathrm{m}$ tangentially negative, reaching the maximum amount of unload for the faulty planet. In the following equation, it is given the absolute rotation value of the carrier between positions of pure radial and tangential components $\left(\frac{\pi}{2}\right.$ or relative rotation of the planet with respect to the carrier).

$$
\begin{gathered}
\theta_{P / \text { carrier }}=\theta_{P}-\theta_{\text {carrier }}= \\
\theta_{\text {carrier }}=0.58 \mathrm{rad}
\end{gathered}
$$

In Figure 23, it can be seen that for the pure negative tangential component and the level of torque used in this simulation $(600 \mathrm{Nm})$, planet 1 becomes load-free and it can also be observed that the LSR curve shape for each of the planets corresponds to that shown in previous sections for negative $e_{\text {tan }}$. From this position, and as the system continues to rotate, the eccentricity error progressively loses tangential component, reaching the maximum positive value of $20 \mu \mathrm{m} e_{\mathrm{rad}}$ at carrier position of $1.16 \mathrm{rad}$. In this position, the LSR also presents a value close to $1 / 3$ due to the lesser effect of the radial positioning error. When the system further rotates, the eccentricity acquires again positive values of $e_{\tan }$ component, showing one more time a LSR behaviour similar to that of a positive tangential pin point positioning error of the planet at $1.74 \mathrm{rad}$. The sinusoidal behaviour of the LSR for the run-out error will repeat itself periodically as the carrier rotates $2.32 \mathrm{rad}$, alternating LSR curve shapes characteristic of $e_{t a n}$ and $e_{\text {rad }}$.

Regarding the transmission error for the planetary transmission with run- 


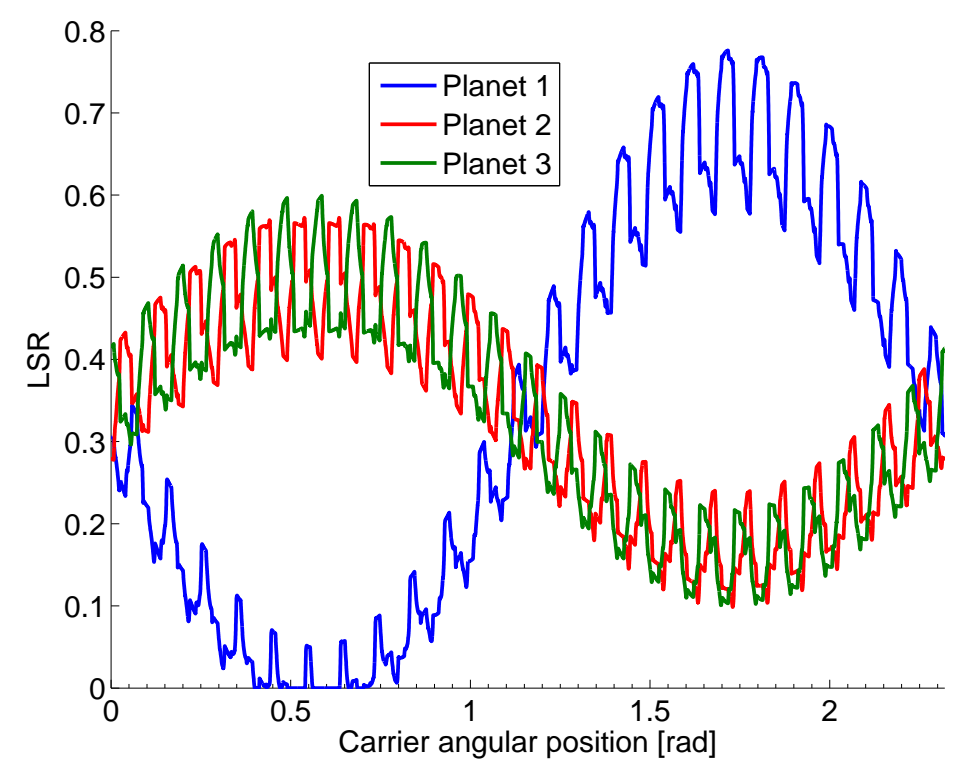

Figure 23: Load sharing ratio with $20 \mu m$ eccentricity error and $600 \mathrm{Nm}$ with a fixed sun configuration

out planet errors, a parallel analysis to that reported for the LSR can be performed. In Figure 24 the same four areas can be identified as the eccentricity changes its dominant component from negative radial to negative tangential to positive radial and positive tangential. Attending to this relatively easily measurable signal, an eccentricity error can thus be identified in a quasi-static analysis.

\subsection{Run-out errors in floating sun configuration}

Similarly to what happened for transmission with positioning error, allowing the sun to move freely causes the absorbtion of the eccentricity effect on the LSR, as shown in Figure 26. Again the variations from the perfect distribution is caused by the loss of symmetry explained before. The orbit described by the sun is presented in Figure 25. The distance to the center depends mainly on the tangential component of the eccentricity for each position, causing the appearance of lobes in the orbit shape.

\section{Conclusions}

Two different planetary configurations have been studied, considering the possibility of both fixed and free translation of the sun and taking into ac- 


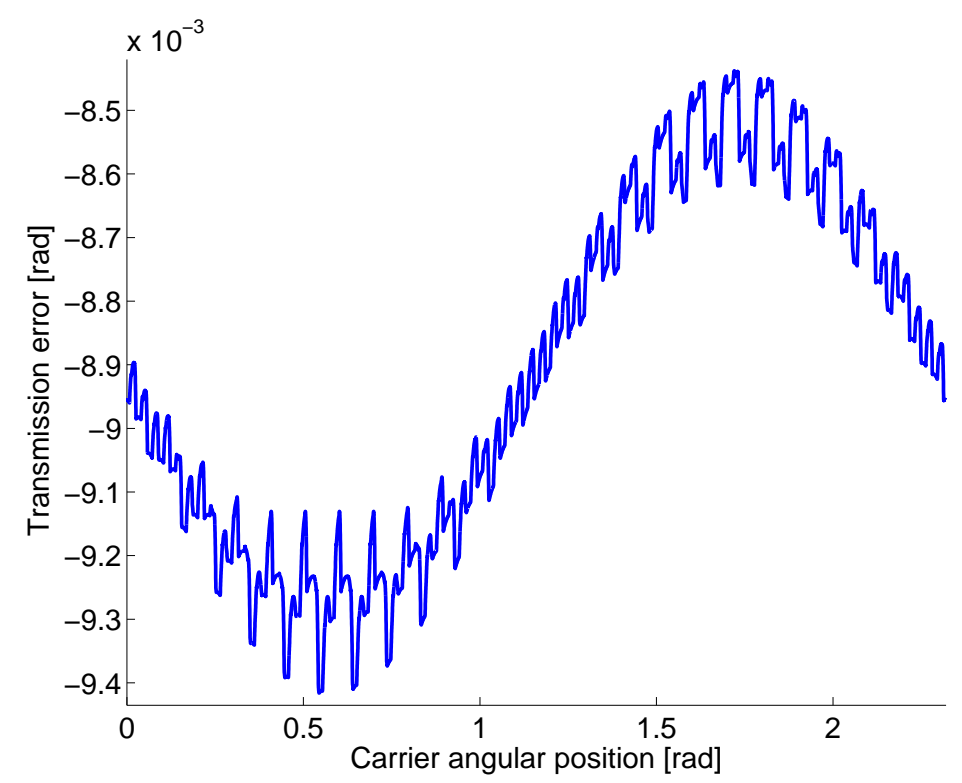

Figure 24: Transmission error with $20 \mu m$ eccentricity error with a fixed sun configuration

count the resulting values of load sharing ratio (LSR) between paths. The results obtained in this paper are valid for any three planet transmission with a $2 \pi / 3$ distribution of the meshing phase, and similar values of operating pressure angles as the ones shown here. The tendency of the load sharing ratio when errors are included can be extrapolated for n-planet transmissions when the configuration is fixed (of course taking into account the proportionality between the number of planets and the ideal LSR and its deviations), but there are critical differences in the behaviour of 3-planet versus n-planet systems when there is a floating central member, as explained in [4]. As expected, the LSR varies widely in the fixed transmission scenario, due to the different path stiffness. These variations on the values of the LSR have been found to have no impact on the maximum level of contact forces, which will not exceed its maximum nominal value in transmissions without defects. In the floating sun scenario, the LSR becomes almost perfect, except for the areas where contacts out of the line of action take place. Nevertheless, even in those zones, the variations of the LSR are insignificant, never above a tenth of percentage point.

Planet positioning errors have been analysed: tangential and radial components in a separately way. The tangential error has a great impact on the 


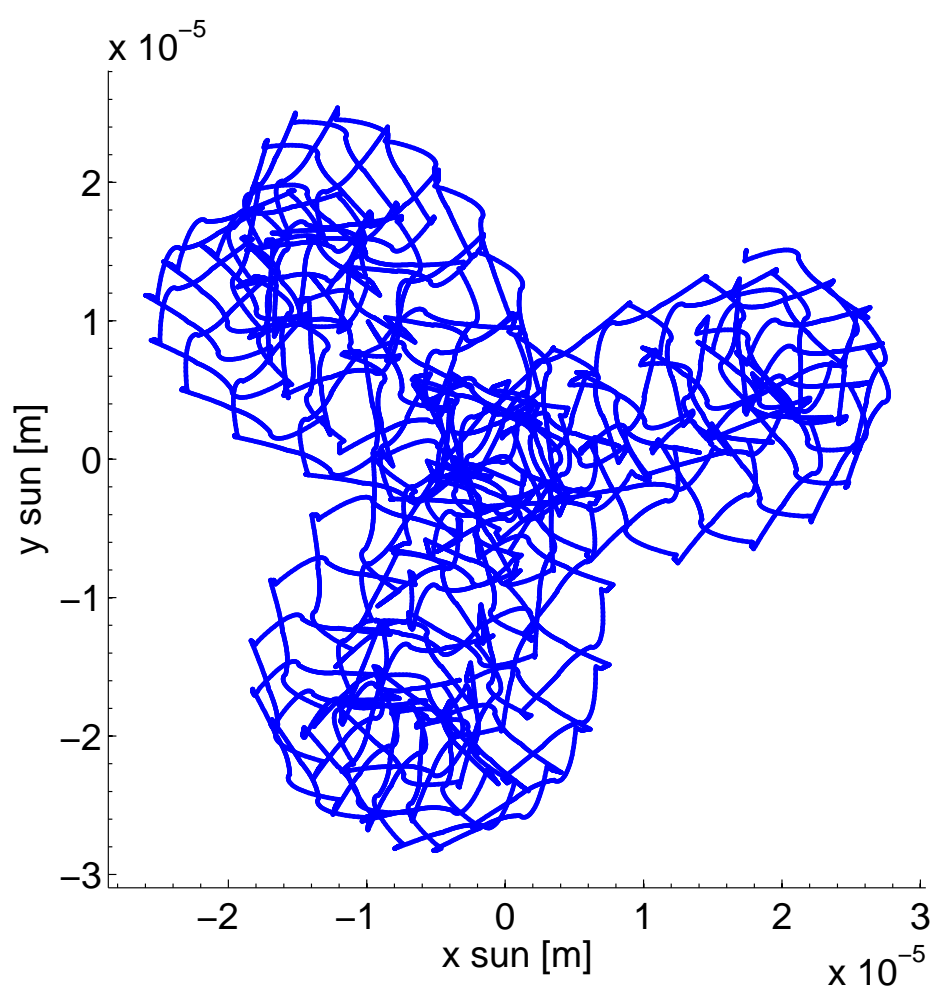

Figure 25: Sun orbit with a floating configuration and $20 \mu m$ eccentricity error

LSR in non floating configurations. It can even cause the complete discharge of one or more paths under certain conditions of deviation and load. Whereas a negative tangential deviation (with respect to the line of action) can cause the unload of the defected planet, a positive deviation has the potential to unload all the non-defected paths. Thus, the consequences of the positive deviations are much more serious than the ones posed by the negative ones.

The effect of the tangential component on the LSR has an impact in the maximum contact forces level. Nevertheless, the relationship between the increase on the contact forces and the variation of the LSR has been found to be nonlinear.

The radial component of the positioning error has a significantly lower impact on the LSR (found to be approximately 40 times lower for the example transmission) than the tangential component. Nonetheless, this impact is not null, in spite of what can be found in some bibliographic references. It strongly depends on the design and mounting conditions of the planetary 


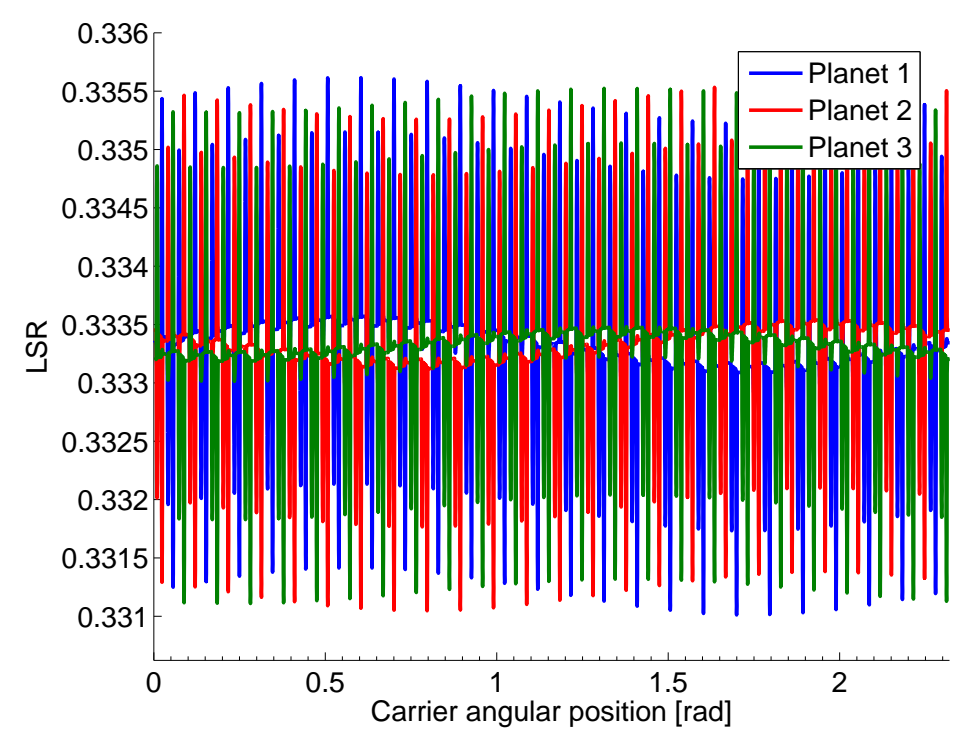

Figure 26: Load sharing ratio with $20 \mu \mathrm{m}$ eccentricity error and $600 \mathrm{Nm}$ with a floating sun configuration

transmission; more specifically on the difference between the pressure angles of sun-planet and planet-ring.

When errors are present, the variations on the values of the LSR have been found to have a much lesser impact on the maximum level of teeth contact forces. Thus, the initial concern that uneven LSR could have great importance on the teeth durability due to higher loads than nominal is partially unfounded.

The incremet of the transmitted load results in the dilution of the positioning error effects, due to the presence of greater levels of global deflections. For the floating configuration, the sun follows an orbit of radius similar to the positioning error magnitude (for tangential components). In this way, the sun displacement absorbs the error and virtually evens the LSR.

The effects of the eccentricity error have also been studied. It has been concluded that they are equivalent with a sinusoidal evolution of the positioning error, through its different components and directions.

Acknowledgments The authors would like to acknowledge Project DPI201344860 funded by the Spanish Ministry of Science and Technology for supporting this research. 


\section{References}

[1] J. D. Smith, Gear Noise and VibrationCited By (since 1996): 95.

[2] T. Hayashi, Y. Li, I. Hayashi, K. Endou, W. Watanabe, Measurement and some discussions on dynamic load sharing in planetary gears, Bull. JSME 29 (253) (1986) 2290-2297, cited By 24.

[3] T. Hidaka, Y. Terauchi, Dynamic behavior of planetary gear - 1. load distributions in planetary gear., Bull JSME 19 (132) (1976) 690-698, cited By 54 .

[4] A. Singh, Load sharing behavior in epicyclic gears: Physical explanation and generalized formulation, Mechanism and Machine Theory 45 (3) (2010) 511-530, cited By 63. doi:10.1016/j.mechmachtheory.2009.10.009.

[5] A. Bodas, A. Kahraman, Influence of carrier and gear manufacturing errors on the static load sharing behavior of planetary gear sets, JSME International Journal, Series C: Mechanical Systems, Machine Elements and Manufacturing 47 (3) (2004) 908-915, cited By 86. doi:10.1299/jsmec.47.908.

[6] F. Oyague, Gearbox modeling and load simulation of a baseline 750-kw wind turbine using state-of-the-art simulation codes, NREL/TP-50041160Cited By 2.

[7] D. Seager, Conditions for the neutralization of excitation by the teeth in epicyclic gearing., J Mech Eng Sci 17 (5) (1975) 293-298, cited By 48.

[8] M. Inalpolat, A. Kahraman, A dynamic model to predict modulation sidebands of a planetary gear set having manufacturing errors, Journal of Sound and Vibration 329 (4) (2010) 371-393, cited By 73. doi:10.1016/j.jsv.2009.09.022.

[9] L. Zhang, Y. Wang, K. Wu, R. Sheng, Q. Huang, Dynamic modeling and vibration characteristics of a two-stage closed-form planetary gear train, Mechanism and Machine Theory 97 (2016) $12-28$. 
[10] G. Liu, R. Parker, Dynamic modeling and analysis of tooth profile modification for multimesh gear vibration, Journal of Mechanical Design, Transactions of the ASME 130 (12) (2008) 1214021-12140213, cited By 44. doi:10.1115/1.2976803.

[11] P. Velex, L. Flamand, Dynamic response of planetary trains to mesh parametric excitations, Journal of Mechanical Design, Transactions of the ASME 118 (1) (1996) 7-14, cited By 96.

[12] J. Lin, R. Parker, Planetary gear parametric instability caused by mesh stiffness variation, Journal of Sound and Vibration 249 (1) (2002) 129145, cited By 146. doi:10.1006/jsvi.2001.3848.

[13] P. Marques, R. Martins, J. Seabra, Power loss and load distribution models including frictional effects for spur and helical gears, Mechanism and Machine Theory 96 (2016) 1-25, cited By 3.

[14] R. Parker, V. Agashe, S. Vijayakar, Dynamic response of a planetary gear system using a finite element/contact mechanics model, Journal of Mechanical Design, Transactions of the ASME 122 (3) (2000) 304-310, cited By 177 .

[15] V. Abousleiman, P. Velex, S. Becquerelle, Modeling of spur and helical gear planetary drives with flexible ring gears and planet carriers, Journal of Mechanical Design, Transactions of the ASME 129 (1) (2007) 95-106, cited By 31. doi:10.1115/1.2359468.

[16] E. Mucchi, G. D'Elia, G. Dalpiaz, Simulation of the running in process in external gear pumps and experimental verification, Meccanica 47 (3) (2012) 621-637, cited By 24. doi:10.1007/s11012-011-9470-9.

[17] F. Chaari, M. Abbes, F. Viadero, A. Fernandez del Rincon, M. Haddar, Analysis of planetary gear transmission in non-stationary operations, Frontiers of Mechanical Engineering 8 (1) (2013) 88-94, cited By 8. doi:10.1007/s11465-013-0361-8.

[18] X. Qiu, Q. Han, F. Chu, Load-sharing characteristics of planetary gear transmission in horizontal axis wind turbines, Mechanism and Machine Theory 92 (2015) 391-406, cited By 1. doi:10.1016/j.mechmachtheory.2015.06.004. 
[19] S. Mo, Y. Zhang, Q. Wu, S. Matsumura, H. Houjoh, Load sharing behavior analysis method of wind turbine gearbox in consideration of multiple-errors, Renewable Energy 97 (2016) 481-491, cited By 0. doi:10.1016/j.renene.2016.05.058.

[20] A. Nejad, Y. Xing, Y. Guo, J. Keller, Z. Gao, T. Moan, Effects of floating sun gear in a wind turbine's planetary gearbox with geometrical imperfections, Wind Energy 18 (12) (2015) 2105-2120, cited By 1. doi:10.1002/we.1808.

[21] A. Fernandez del Rincon, F. Viadero, M. Iglesias, P. Garcia, A. De-Juan, R. Sancibrian, A model for the study of meshing stiffness in spur gear transmissions, Mechanism and Machine Theory 61 (2013) 30-58, cited By :43.

[22] A. Fernandez del Rincon, M. Iglesias, A. De-Juan, P. Garcia, R. Sancibrian, F. Viadero, Gear transmission dynamic: Effects of tooth profile deviations and support flexibility, Applied Acoustics 77 (2014) 138-149, cited By :17.

[23] M. Iglesias, A. Fernandez del Rincon, A. de Juan, A. Diez-Ibarbia, P. Garcia, F. Viadero, Advanced model for the calculation of meshing forces in spur gear planetary transmissions, Meccanica 50 (7) (2015) 1869-1894, cited By 0. doi:10.1007/s11012-015-0130-3.

[24] E. Mucchi, G. Dalpiaz, A. Rivola, Elastodynamic analysis of a gear pump. part ii: Meshing phenomena and simulation results, Mechanical Systems and Signal Processing 24 (7) (2010) 2180-2197.

[25] A. Diez-Ibarbia, A. Fernandez del Rincon, M. Iglesias, A. De-Juan, P. Garcia, F. Viadero, Efficiency analysis of spur gears with a shifting profile, Meccanica 51 (3) (2016) 707-723.

[26] A. Singh, Load sharing behavior in epicyclic gears: Physical explanation and generalized formulation, Mechanism and Machine Theory 45 (3) (2010) 511-530, cited By 63. doi:10.1016/j.mechmachtheory.2009.10.009.

[27] A. Singh, Epicyclic load sharing map - development and validation, Mechanism and Machine Theory 46 (5) (2011) 632-646, cited By 29. doi:10.1016/j.mechmachtheory.2011.01.001. 
[28] X. Gu, P. Velex, On the dynamic simulation of eccentricity errors in planetary gears, Mechanism and Machine Theory 61 (2013) 14-29, cited By 18. doi:10.1016/j.mechmachtheory.2012.10.003. 